\title{
Subband population and electron subband mobility for two interacting Si- $\delta$-doping layers in GaAs
}

\author{
P.M. Koenraad ${ }^{a}$, A.C.L. Heessels ${ }^{a}$, F.A.P. Blom ${ }^{a}$, J.A.A.J. Perenboom ${ }^{b}$ and \\ J.H. Wolter ${ }^{a}$ \\ ${ }^{a}$ Department of Physics, Eindhoven University of Technology, The Netherlands \\ ${ }^{b}$ High Field Magnet Laboratory, University of Nijmegen, The Netherlands
}

\begin{abstract}
In this paper we present measurements of the subband population and quantum mobility in the various subbands of GaAs samples that contain two coupled $\mathrm{Si}$ - $\delta$-layers and of GaAs samples that contain a single $\delta$-doping layer which was increased in thickness by thermal annealing. The measured subband population will be compared with the subband population obtained from self-consistent solutions of the coupled Poisson and Schrödinger equation. The experimental results on both types of structures are compared and show that the population of the higher subbands is not sensitive to the charge distribution of the ionized donors in the center of the confining potential. The quantum mobility in the highest subbands on the contrary is sensitive to the distribution of the ionized donors.
\end{abstract}

\section{Introduction}

With growth techniques like Molecular Beam Epitaxy (MBE) and Chemical Beam Epitaxy (CBE) it is possible to control the concentration and doping profile at an atomic level. With present-day techniques one can easily obtain doping profiles with a thickness of $20 \AA$ or less by growing at $480^{\circ} \mathrm{C}$ or less. Because the width of the resulting confining potential of the ionized donors is in the order of $200 \AA$, quantum states are formed in the potential well. When the donor concentration in the doping layer is larger than $0.3 \times 10^{12}$, metallic conduction occurs in the layer and thus a degenerate 2-dimensional electron gas is formed. Normally more than one subband is populated in these structures. The charge distribution of the electrons over the subbands is determined by the width of the doping profile.

The mobility of the electrons in $\delta$-doped structures is very low compared to GaAs/

Correspondence to: P.M. Koenraad, Physics Department, Eindhoven University of Technology, P.O. Box 513, 5600 MB Eindhoven.
$\mathrm{Al}_{x} \mathrm{Ga}_{1-x} \mathrm{As}$ heterostructures because of the strong overlap between the electrons and the ionized donors in $\delta$-doped structures. Due to the fact that the wavefunction in each subband is different the overlap of the wavefunction with the ionized donors will not be the same in each subband. Thus the mobility also differs for each subband

In this paper we have studied the subband population in GaAs samples containing two $20 \AA$ thick $\mathrm{Si}-\delta$-doping layers in close proximity of another. Our self-consistent calculations show that coupling between the two layers is still strong in structures where the separation is $200 \AA$. We have determined the coupling between the two $\delta$-doped layers from the subband population determined by Shubnikov-de Haas ( $\mathrm{SdH}$ ) measurements. The effect of the coupling between the $\delta$-layers on the mobility of the electrons in the various subbands was also studied by determination of the quantum mobility from the $\mathrm{SdH}$ oscillations.

These results on subband population and mobility will be compared with results obtained on GaAs structures which contained only one $\delta$-doping spike. The width of the confining 
potential in this single $\delta$-doped structure was increased by thermal annealing. From the comparison of the subband population and the mobility in both type of structures we can determine the sensitivity of the electric transport properties on the distribution of the ionized donors.

Kido et al. [1] also studied GaAs structures containing two adjacent Si- $\delta$-doping in GaAs. They found that the subband population in their double doped structures was equal to the subband population of a $\mathrm{GaAs}$ structure containing a single thick $\mathrm{Si}-\delta$-layer.

\section{Theory}

We calculated the confining potential, subband energies and envelope wavefunctions self-consistently by solving simultaneously the coupled Poisson and Schrödinger equation. Due to the high electron density in a $\delta$-doped structure more than one subband is populated. Therefore we have to solve the following one-dimensional Shrödinger equation for each subband separately:

$\left\{-\frac{\hbar^{2}}{2 m^{*}} \frac{\partial^{2}}{\partial z^{2}}+U(z)\right\} \psi_{i}(z)=E_{i} \psi_{i}(z)$

where $i$ is the subband index. The potential $U(z)$ consists of only one term in the case of $\delta$-doped structures and is given by the Poisson equation

$\frac{\partial^{2}}{\partial z^{2}} U(z)=\frac{e \rho(z)}{\varepsilon_{0} \varepsilon_{\mathrm{r}}}$

where $\rho(z)$ is the charge distribution which is the sum of the ionized donors, the electrons and the charged background impurities. The equations are coupled because $\psi_{i}(z), U(z)$ and $E_{i}$ appear in both equations. The nonparabolicity of the $\Gamma$ conduction is taken into account in these calculations. The exchange and correlation potential can be neglected in $\delta$-doped structures with high doping concentrations [2].

Figure 1 shows the probability distribution $\left|\psi_{i}(z)\right|^{2}, E_{i}$ and $U(z)$ in a $\delta$-doped structure that contains $2.5 \times 10^{12} \mathrm{~cm}^{-2}$ electrons, two $\delta$-doping

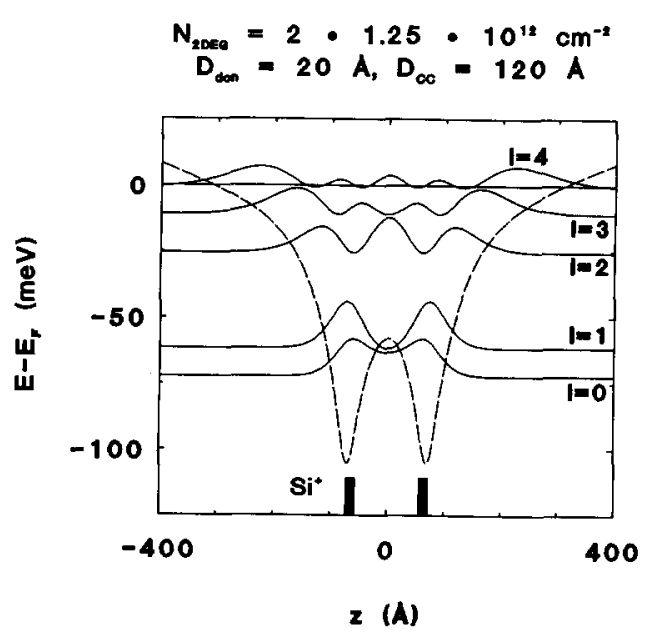

Fig. 1. The probability distribution of the electrons in the various subbands (solid line) and the electrostatic potential (dashed line) in a GaAs structure containing two adjacent Si- $\delta$-layers. The total electron density is $2.5 \times 10^{12} \mathrm{~cm}^{-2}$ and the donor concentration is equal in the two doping layers that are separated by $120 \AA$.

layers of $20 \AA$ thick separated at $120 \AA$ and a background impurity concentration of $10^{15} \mathrm{~cm}^{-3}$. The figure clearly shows that the two $\delta$-layers give rise to a single confining potential.

In fig. 2 we have plotted the subband energy in the same structure as described above as a function of the separation of the doping layers. The dependence of the subband energy on the dis-

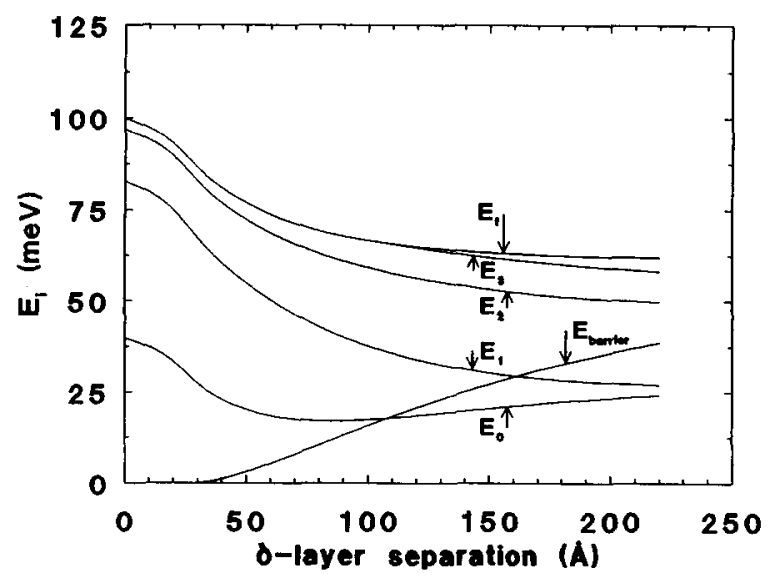

Fig. 2. The subband energy in a double Si- $\delta$-doped GaAs structure with an electron density of $2.5 \times 10^{12} \mathrm{~cm}^{-2}$ as a function of the separation between the two doping layers. $E_{\text {barrier }}$ is the barrier height between the two $\delta$-doping layers. 
tance clearly resembles the dependence of the energy levels in two coupled quantum wells as described by Merzbacher [3]. If the double well would describe a di-atomic molecule then the even subbands would correspond to the bonding states and the odd states to the anti-bonding states. At a separation of $220 \AA$ we observe that the $i=0$ and $i=1$ subbands have become almost degenerate. Another way to represent this degeneracy is by defining the wavefunctions

$$
\Psi_{1}(z)=\left[\psi_{0}(z)+\psi_{1}(z)\right] / \sqrt{2}
$$

and

$$
\Psi_{\mathrm{r}}(z)=\left[\psi_{0}(z)-\psi_{1}(z)\right] / \sqrt{2}
$$

which correspond to the electrons trapped in the left or the right potential well of the $\delta$-doping layer, respectively.

\section{Experiments}

The samples were grown at $480^{\circ} \mathrm{C}$ in a computer-controlled Varian MBE system. We grew GaAs samples containing two Si- $\delta$-layers separated by $20 \AA, 40 \AA, 80 \AA, 120 \AA$ and $160 \AA$, respectively. The intended doping concentration was $2.5 \times 10^{12} \mathrm{~cm}^{-2}$ per $\delta$-layer. We also grew two samples containing either a single doping layer of $2.5 \times 10^{12} \mathrm{~cm}^{-2}$ or $5 \times 10^{12} \mathrm{~cm}^{-2}$. These structures correspond to the cases of infinite separation of the $\delta$-layers and no separation of the $\delta$-layers, respectively. In order to obtain a planar doping profile on a smooth surface the growth of the GaAs was interrupted by closing the $\mathrm{Ga}$ shutter $10 \mathrm{~s}$ before opening the $\mathrm{Si}$ furnace.

The structure with a single doping layer of $5 \times 10^{12} \mathrm{~cm}^{-2}$ was used in the annealing experiments. A number of samples from this wafer were annealed in a Rapid Thermal Annealer (RTA) at $800^{\circ} \mathrm{C}$ for $3 \mathrm{~s}, 10 \mathrm{~s}, 30 \mathrm{~s}, 100 \mathrm{~s}$ and $300 \mathrm{~s}$, respectively. The samples were annealed face down on a Si-wafer in a controlled $\mathrm{N}_{2}$ atmosphere.

The subband population can be determined from Shubnikov-de Haas measurements. We carried out measurements on Hall bar shaped samples in magnetic fields up to $20 \mathrm{~T}$. The weak oscillations in $\rho_{x x}$ and $\rho_{x y}$ were resolved by measuring $\mathrm{d} \rho_{x x} / \mathrm{d} B$ and $\mathrm{d} \rho_{x y} / \mathrm{d} B$ with a modulation field of $18 \mathrm{mT}$ at a frequency of $21.3 \mathrm{~Hz}$. The subband population was determined from the Fourier transform of $\mathrm{d} \rho_{x x} / \mathrm{d} B$ and $\mathrm{d} \rho_{x y} / \mathrm{d} B$. The doping concentration in all the samples with a double $\delta$-layer was close to the intended doping concentration of $5 \times 10^{12} \mathrm{~cm}^{-2}$. In the two samples with a single $\delta$-doping layer we found that the width of the doping profile was equal to $20 \AA$ or less.

The $\mathrm{SdH}$ oscillations of a single subband can be separated by filtering the corresponding peak in the frequency domain of the Fourier transform. The SdH oscillations of a single subband can then be obtained by taking the inverse Fourier transform of this peak. The quantum or single-particle mobility of such a single subband was determined from a so-called Dingle plot [4]. In such a plot the logarithmetic amplitude of the $\mathrm{SdH}$ oscillations is plotted against $1 / B$. The slope of the straight line through the data points is equal to $\pi / \mu_{q}$. Normally for each subband we have at least 6 to 8 data points to determine the quantum mobility. The accuracy for the quantum mobility is about $10 \%$ in this case. When the electron concentration in a subband is smaller than $0.3 \times 10^{12} \mathrm{~cm}^{-2}$ the number of data points reduces to 2 or 3 and thus the accuracy will be less.

\section{Discussion}

In fig. 3(A) we have plotted the measured subband population as a function of the distance between the doping layers. In the same figure we also show the calculated subband population as determined by the self-consistent calculations. We observe a very nice accordance between theory and experiment. The structure with the doping layers $160 \AA$ apart showed only one peak in the high-density region of the Fourier transform. This single peak actually is the overlap of the two peaks of the $i=0$ and $i=1$ levels which have become degenerate at this distance of the 


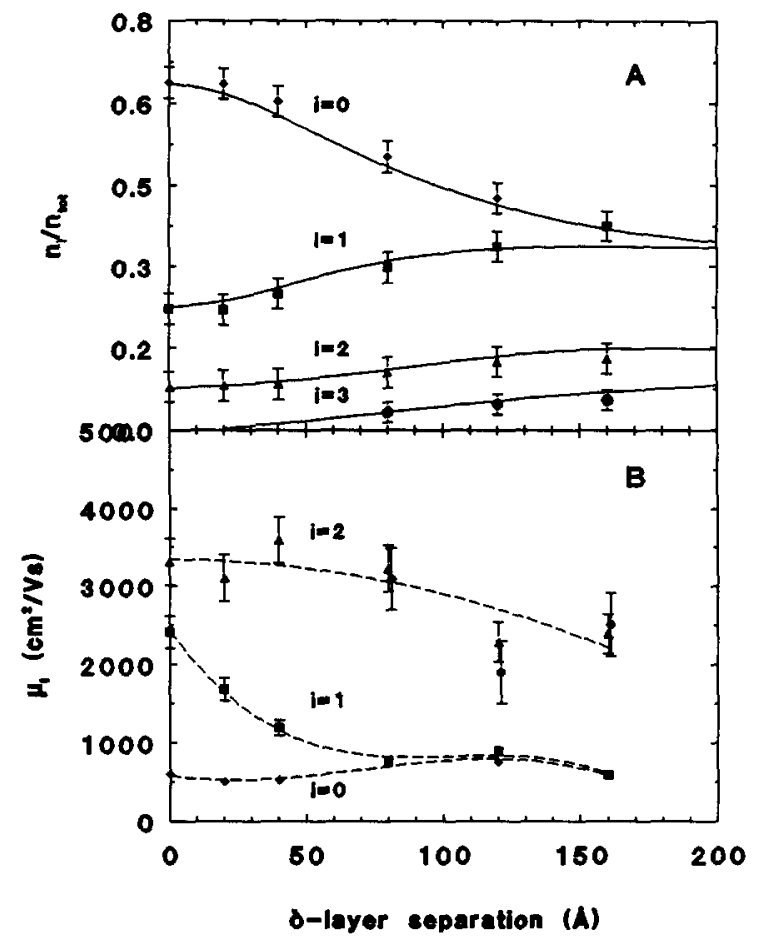

Fig. 3. (A) Subband population in the double $\delta$-doped structure. The solid line represents the calculated subband population. (B) Quantum subband mobility in the double $\delta$-doped structure. The dashed line is only to guide the eye.

doping layers. This result is contrary to the results of Kido et al. [1] who conclude that two adjacent $\delta$-layers behave, up to distances of $300 \AA$, as a single doping layer.

In fig. 4(A) we show the subband population in the annealed samples as a function of the thickness of the doping layer. The thickness of the doping layer was determined from the fit of the measured subband population to subband population as obtained from calculations where we used the doping profile thickness as the free parameter. The thickness we give is the fullwidth-half-maximum of the Gauss curve describing the doping profile. The figure clearly shows that we do not observe any degenerate levels in the annealed sample. This was of course to be expected for an uncoupled $\delta$-layer.

Comparisons of the measured subband population in both types of structures (see fig. 5) shows that the population in the $i=1, i=2$ and

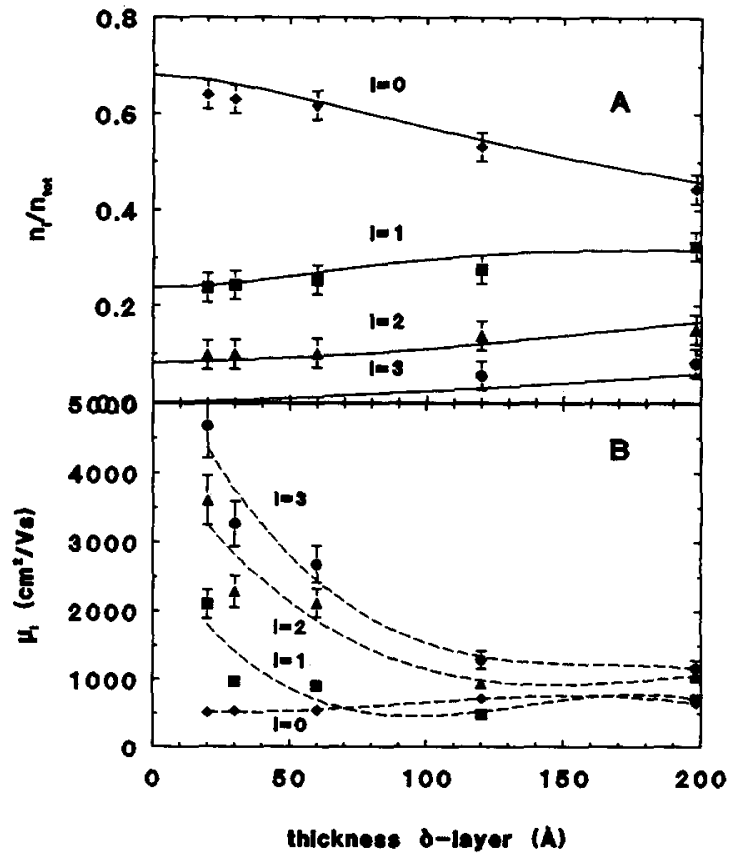

Fig. 4. (A) Subband population in the annealed structure containing a single doping layer. The solid line represents the calculated subband population. (B) Quantum subband mobility in the annealed structure containing a single doping layer. The dashed line is only to guide the eye.

$i=3$ subband is almost equal for the single and double $\delta$-doped structures. The difference is only found in the $i=0$ level. This implies that for the higher levels it does not matter what the potential shape in the center of the potential well looks like. Thus the subband population of the higher levels is not sensitive to the charge distribution in the center of the well. It is very appealing that we find the same subband population for the higher subbands in a single-doped structure and a double-doped structure where the distance between the two adjacent doping profiles is equal to full-width-half-maximum of the Gaussian profile in the single-doped structure.

In fig. 3(B) we show the quantum mobility in the subbands for the structure with two adjacent doping layers. When the two doping layers are separated enough we observe that the mobility in the $i=0$ and the $i=1$ subband become equal. This is to be expected when the two levels 


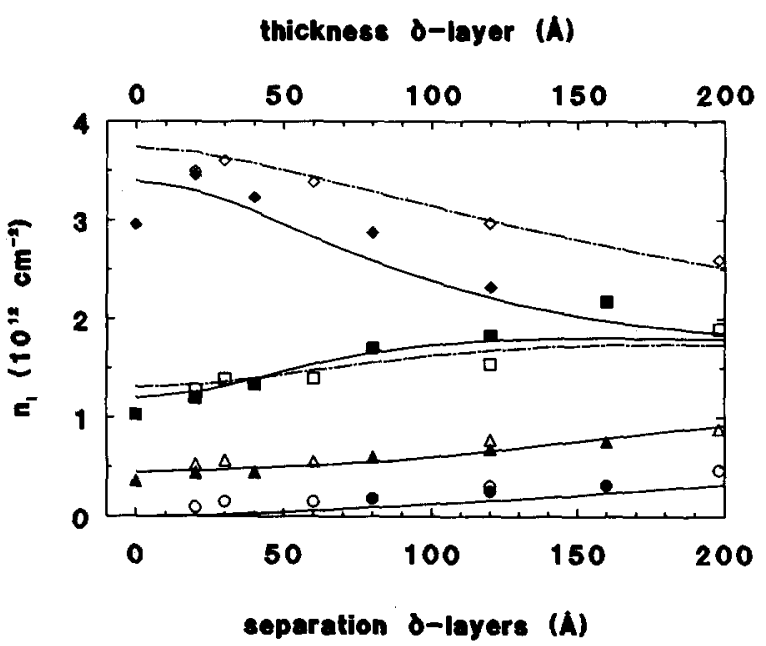

Fig. 5. The subband population in the structure containing two doping layers (open symbols) and in the annealed structure containing a single doping layer (filled symbols). The lines represent the calculated subband population. The top axis gives the FWHM of the Gaussian doping profile in the annealed sample. The bottom axis gives the separation of the two doping layers in the double-doped structure.

become degenerate. The quantum mobility in the $i=2$ and to some extent (not very accurate) also the $i=3$ subband shows a decreasing mobility when the doping layers are separated.

In fig. 4(B) the mobility as a function of the thickness of the doping layer is shown. It is evident that the mobility in all subbands rapidly decreases to the $i=0$ value for all the subbands. In the samples with the thickest doping profile the quantum mobility is the same in all subbands.

Comparing again the results on the single $\delta$ doped and double $\delta$-doped structure we see that in the double-doped structure the mobility in the $i=0$ and $i=1$ subbands decreases comparable to the mobility in the annealed single $\delta$-doped structures. But the mobility in the $i=2$ and $i=3$ subbands does not show such a rapid decrease as the annealed samples. This comparison shows that contrary to the subband population of the higher subbands the quantum subband mobility in these subbands is sensitive to the actual doping profile in the center of the well.

\section{Conclusions}

Our measurements show that in samples with two doping layers $20 \AA$ thick and a doping concentration of $2.5 \times 10^{12} \mathrm{~cm}^{-2}$ the $i=0$ and $i=1$ subband become degenerate at a separation of $160 \AA$. The measurements also show that the subband population of the higher levels is not sensitive to the charge distribution because we observe the same subband population for the $i=1, i=2$ and $i=3$ levels in samples with the doping either distributed over two doping layers or with one central doping spike. Contrary to the subband population the subband quantum mobility in $i=2$ and $i=3$ is dependent on the distribution of the ionized donors.

\section{Acknowledgements}

We kindly thank M.R. Leys and W.C. van der Vleuten for the sample growth, I. Barsony of the University of Twente for annealing the samples, and P. Nouwens for the sample preparation.

\section{References}

[1] G. Kido, S. Yamada and T. Makimoto, Physica B 177 (1992) 433.

[2] P.M. Koenraad, F.A.P. Blom, C.J.G.M. Langerak, M.R. Leys, J.A.A.J. Perenboom, J. Singleton, S.J.R.M. Spermon, W.C. van der Vleuten, A.P.J. Voncken and J.H. Wolter, Semicond. Sci. Technol. 5 (1990) 861.

[3] E. Merzbacher, Quantum Mechanics (Wiley, New York, 1961) p. 65.

[4] P.M. Koenraad, B.F.A. van Hest, F.A.P. Blom, R. van Dalen, M. Leys, J.A.A.J. Perenboom and J.H. Wolter, Physica B 177 (1992) 485. 